\title{
IMPORTANCIA DE LA EXPERIENCIA Y DE LA VARIACIÓN INTERMEDIDOR EN LA TOMA DE MEDIDAS CRANEALES PARA ESTUDIOS MORFOMÉTRICOS
}

\author{
ANTONIO SANTOS-MORENO', SALVADOR GAONA ${ }^{2}$ \\ Y YOLANDA HORTELANO'
}
${ }^{1}$ Departamento de Zoología, Instituto de Biología, UNAM, Apartado Postal 70-153, México, D.F., 04510.
${ }^{2}$ Departamento de Biología, UAM-Iztapalapa, Apartado Postal 55-535, México. D.F., 09340.

Palabras clave: Variación, error de medición, morfometría, Reithrodontomys sumichrasti.

Una gran cantidad de estudios ecológicos, evolutivos y principalmente sistemáticos basan sus conclusiones total o parcialmente en la inferencia del análisis de caracteres merísticos (Lee, 1990). Así mismo los datos morfométricos de variables medidas en una escala continua, son importantes en la generación y confirmación de hipótesis evolutivas y jerarquías taxonómicas (Bailey y Byrnes, 1990). A pesar de esto, la medición de ejemplares para estudios morfométricos es comúnmente asignada a personas sin entrenamiento, asumiéndose que es un factor sin relevancia en los resultados.

De los pocos precedentes de este tipo de estudios se puede mencionar el trabajo de Lee (1982) quien encontró que la variabilidad observada en caracteres cuantitativos de renacuajos está fuertemente correlacionada con la precisión con la que son medidos. En otro estudio (Lee, 1992) cuantificó la variación intraobservador en el conteo de escamas en lagartijas del género Anolis, encontrando diferencias hasta de un orden de magnitud.

Asimismo Santos Moreno (1994) al analizar la variabilidad debida a distintos medidores en caracteres externos estándar en Peromyscus melanotis, encontró diferencias significativas en una de cuatro (longitud de la cola) y que más del $13 \%$ de la variabilidad en la muestra era asignable a diferencias en el medidor. En este último trabajo las medidas analizadas pertenecían a distintos ejemplares, por lo que éstas diferencias eran el resultado tanto de la variación intermedidor como de la variación intrapoblacional. Dada la importancia de este tipo de variación y que en el caso de mamíferos no existen muchos estudios encaminados a cuantificarla, el objetivo de este trabajo es analizar la variabilidad debida a la experiencia y a las diferencias entre medidores en la toma de datos morfométricos craneales, así como estimar números mínimos de ejemplares requeridos para obtener estimaciones confiables 
estadísticamente de las medias de caracteres de uso común en estudios morfométricos.

Se tomaron 20 medidas craneales, comunes en estudios morfométricos de mamíferos, en 15 ejemplares de Reithrodontomys sumichrasti, por tres personas: una con experiencia previa en la toma de éstas medidas (Medidor 1) y dos más sin experiencia (Medidores 2 y 3), a todos se les proporcionó un esquema ilustrando las medidas e instrucciones detalladas de cómo tomarlas. Cada persona midió los ejemplares en tres ocasiones, registrando independientemente cada grupo de medidas, para evitar posibles sesgos. Los ejemplares corresponden a cuatro localidades de los Estados de Puebla y Veracruz, México y se encuentran depositados en la Colección Mastozoológica de la Universidad Autónoma Metropolitana-Iztapalapa.

Las medidas consideradas y las abreviaturas con que se les menciona posteriormente en el texto, figura y cuadros son las siguientes (Figura 1): Longitud máxima del cráneo (LOCR), longitud condilobasal (LOCO), longitud del paladar (LOPA), longitud de los nasales (LONA), longitud rostral (LORO), anchura anterior de los nasales (ANNA), anchura posterior de los nasales (ANNAP), anchura cigomática (ANCI), anchura de la caja craneal (ANCC), anchura interorbital (ANIN), anchura de los frontales (AMF), altura de la caja craneal (ALCR), longitud de la
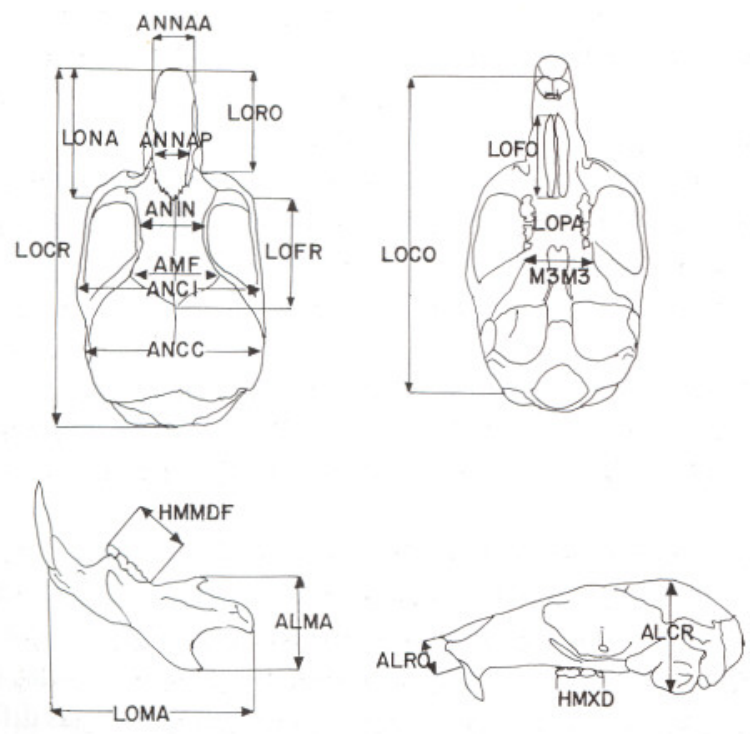

Figura 1. 20 medidas tomadas en cráneos de Reithrodontomys sumichrasti. Ver abreviaturas en el texto. 
mandíbula (LOMA), altura de la mandíbula (ALMA), longitud de la hilera mandíbular de dientes (HMMDF), longitud de la hilera maxilar de dientes (HMXD), altura rostral (ALRO), anchura entre los terceros molares superiores (M3M3), longitud de los frontales (LOFR) y longitud del foramen palatino (LOFO). Estas medidas se tomaron con un vernier electrónico con una precisión de $0.01 \mathrm{~mm}$ (ninguna medida se tomó con lupa).

Se analizó la variación debida a la experiencia tanto en las medias como en las varianzas para cada Medidor. Para el primer caso, se aplicaron análisis de varianza modelo II entre repeticiones por Medidor, y se aplicó la prueba de Levene (Manly, 1986) para estudiar la homogeneidad de varianzas entre los tres grupos de medidas, así como también la proporción de varianza debida a variación entre repeticiones para cada persona y entre Medidores. Los niveles de significancia en todas las pruebas se ajustaron con la prueba secuencial de Bonferroni (Rice, 1989).

A partir del primer grupo de mediciones de cada persona se calculó el tamaño muestral mínimo requerido para intervalos de confianza del $95 \%$, con una anchura del $5 \%$ de la desviación estándar de cada variable modificando la formula de Daniel (1982) $N=z^{2} v a r /(d)^{2}$, donde $z$ corresponde al área bajo la curva de la distribución normal a un nivel de confianza determinado; en este caso el $95 \%$, pero debido al tamaño muestral pequeño, se empleo el valor del área bajo la curva de la distribución t-student (1.753); var es la varianza y $d$ es la anchura deseada del intervalo. Se seleccionó un $5 \%$ del valor de la media de cada variable. Con este mismo conjunto de datos se analizó la variación intermedidor, empleándose las mismas técnicas estadísticas que para el caso de la variación debida a la experiencia.

Para el medidor con experiencia no se encontraron diferencias ni en medias ni en varianzas entre las repeticiones (Cuadro 1). En contraste, para los medidores sin experiencia si hubo variaciones significativas: en la variable ANCC tanto medias como varianzas difirieron significativamente para el segundo medidor, mientras que para el tercero la variable ANNAA también difirió significativamente en ambos aspectos y ANNAP sólo en varianzas. La proporción de varianza debida a la repetición (Cuadro 1) fue de 0.225 (LOCO) a $41.3 \%$ (ANNAP) para el medidor con experiencia, de 0.42 (LOCR) a $47.9 \%$ (ANCC) para el segundo medidor y de 3.3 (ANCI) a $48.2 \%$ (ANNAA) el tercero.

Cuando se comparó la primera serie de cada medidor (Cuadro 3), el porcentaje de varianza asignable a diferencias entre medidores fluctuó entre 18.9 (LOCR) y $49.2 \%$ (ANNAA). Al comparar las medias entre medidores para la primera repetición, las variables LORO, ANNAA y ANNAP (Cuadro 3) difirieron significativamente en medias y sólo una (ANNAP) fue significativamente heterocesdástica. Los tamaños muestrales requeridos para intervalos del $95 \%$ de confianza (Cuadro 2), variaron considerablemente: De seis (HMXD, Medidor 2) a 124 (ANIN, Medidor 2), con promedio de 26 ejemplares. 
Cuadro 1. Comparación de varianzas (línea superior) y de medias (línea inferior) entre repeticiones por medidor, así como porcentaje de varianza explicada por la diferencia entre repeticiones (\%VAR). $\mathrm{F}=$ valor del estadístico $\mathrm{F}, \mathrm{P}>\mathrm{F}=$ nivel de significancia. $\mathrm{El}$ asterisco indica $\mathrm{P}<0.05$ para la prueba secuencial de Bonferroni.

Medidor 1

Medidor 2

Medidor 3

\begin{tabular}{|c|c|c|c|c|c|c|c|}
\hline Variable & $\mathrm{F}$ & $\mathrm{P}>\mathrm{F} \quad \% \mathrm{VAR}$ & $\mathrm{F}$ & $\mathrm{P}>\mathrm{F} \quad \% \mathrm{VAR}$ & $\mathrm{F}$ & $\mathrm{P}>\mathrm{F}$ & $\%$ VAR \\
\hline \multirow[t]{2}{*}{ LOCR } & 0.07 & 0.92897 .14 & 0.02 & 0.98060 .42 & 0.1 & 0.9088 & 7.309 \\
\hline & 0.08 & 0.9201 & 0 & 0.9957 & 0.09 & 0.9181 & \\
\hline \multirow[t]{2}{*}{$\mathrm{LOCO}$} & 0.03 & 0.97170 .255 & 0.03 & 0.969518 .082 & 0.93 & 0.4038 & 39.523 \\
\hline & 0 & 0.9974 & 0.28 & 0.7548 & 1.89 & 0.1643 & \\
\hline \multirow[t]{2}{*}{ LOPA } & 1.05 & 0.359524 .016 & 0.12 & 0.883510 .988 & 0.14 & 0.8721 & 6.361 \\
\hline & 0.46 & 0.6331 & 0.14 & 0.869 & 0.07 & 0.9298 & \\
\hline \multirow[t]{2}{*}{ LONA } & 0.17 & 0.84071 .963 & 0.17 & 0.84194 .303 & 0.15 & 0.8597 & 8.938 \\
\hline & 0.02 & 0.9798 & 0.05 & 0.9541 & 0.11 & 0.8971 & \\
\hline \multirow[t]{2}{*}{ LORO } & 0.36 & 0.70095 .138 & 1.55 & 0.223437 .826 & 0.22 & 0.801 & 18.903 \\
\hline & 0.06 & 0.9444 & 1.55 & 0.2234 & 0.3 & 0.7395 & \\
\hline \multirow[t]{2}{*}{ ANNAA } & 0.01 & 0.991634 .502 & 0.33 & 0.719842 .44 & 12.35 & $0.0001 *$ & 48.182 \\
\hline & 1.11 & 0.338 & 2.81 & 0.0717 & 13.26 & $0.0001^{*}$ & \\
\hline \multirow[t]{2}{*}{ ANNAP } & 0.1 & 0.906641 .25 & 2.45 & 0.098943 .092 & 7.48 & $0.0017^{*}$ & 45.818 \\
\hline & 2.36 & 0.1071 & 3.12 & 0.0546 & 5.48 & 0.0077 & \\
\hline \multirow[t]{2}{*}{ ANCI } & 0.03 & 0.96590 .442 & 0.96 & 0.392423 .074 & 0.04 & 0.9625 & 3.258 \\
\hline & 0 & 0.9955 & 0.43 & 0.6543 & 0.03 & 0.9658 & \\
\hline \multirow{2}{*}{$\begin{array}{l}\text { ANCC } \\
9.993\end{array}$} & 0.22 & 0.912 & 9.65 & $0.0004^{*}$ & 47.911 & 0.12 & 0.8831 \\
\hline & 0.01 & 0.9907 & 11.47 & $0.0001^{*}$ & & 0.12 & 0.8829 \\
\hline \multirow[t]{2}{*}{ ANIN } & 0.09 & 0.9157 .618 & 4.3 & $0.02 \quad 45.646$ & 0.24 & 0.7914 & 5.464 \\
\hline & 0.09 & 0.9142 & 5.24 & 0.0093 & 0.06 & 0.9405 & \\
\hline \multirow[t]{2}{*}{ AMF } & 0.11 & 0.89862 .89 & 1.11 & 0.339343 .622 & 1.08 & 0.35 & 43.402 \\
\hline & 0.03 & 0.9698 & 3.42 & 0.0421 & 3.29 & 0.0471 & \\
\hline \multirow[t]{2}{*}{ ALCR } & 0.21 & 0.80928 .611 & 0.21 & 0.81234 .263 & 0.65 & 0.5254 & 35.225 \\
\hline & 0.1 & 0.9014 & 1.09 & 0.346 & 1.19 & 0.3137 & \\
\hline \multirow[t]{2}{*}{ LOMA } & 0.08 & 0.925213 .583 & 2.21 & 0.122536 .297 & 0.33 & 0.7182 & 6.011 \\
\hline & 0.19 & 0.8305 & 1.32 & 0.2768 & 0.07 & 0.9341 & \\
\hline \multirow[t]{2}{*}{ ALMA } & 0.17 & 0.84190 .822 & 0.1 & 0.90616 .258 & 2.35 & 0.108 & 18.576 \\
\hline & 0.01 & 0.9917 & 0.07 & 0.9311 & 0.3 & 0.7456 & \\
\hline \multirow[t]{2}{*}{ HMMDF } & 0.09 & 0.91861 .091 & 1.49 & 0.236926 .995 & 1.36 & 0.2678 & 29.822 \\
\hline & 0.01 & 0.9889 & 0.59 & 0.5606 & 0.74 & 0.4837 & \\
\hline \multirow[t]{2}{*}{ HMXD } & 0.45 & 0.64234 .011 & 1.31 & 0.279937 .17 & 1.38 & 0.2632 & 24.274 \\
\hline & 0.04 & 0.9574 & 1.45 & 0.2464 & 0.47 & 0.6271 & \\
\hline
\end{tabular}


Cuadro 1. Continuación...

Medidor 1

Medidor 2

Medidor 3

\begin{tabular}{|c|c|c|c|c|c|c|c|}
\hline Variable & $\mathrm{F}$ & $\mathrm{P}>\mathrm{F} \quad \% \mathrm{VAR}$ & $\mathrm{F}$ & $\mathrm{P}>\mathrm{F} \quad \% \mathrm{VAR}$ & $\mathrm{F}$ & $\mathrm{P}>\mathrm{F}$ & $\%$ VAR \\
\hline \multirow[t]{2}{*}{ ALRO } & 0.2 & 0.818820 .158 & 1.42 & 0.253844 .447 & 0.66 & 0.5246 & \multirow[t]{2}{*}{11.448} \\
\hline & 0.34 & 0.7153 & 4 & 0.0256 & 0.15 & 0.8625 & \\
\hline \multirow[t]{2}{*}{ M3M3 } & 0.16 & 0.855732 .291 & 0.23 & 0.796325 .479 & 0 & 0.9978 & \multirow[t]{2}{*}{38.844} \\
\hline & 0.91 & 0.4096 & 0.52 & 0.5986 & 1.74 & 0.1878 & \\
\hline \multirow[t]{2}{*}{ LOFR } & 1.84 & 0.171136 .888 & 0.16 & 0.84979 .991 & 0.35 & 0.7086 & \multirow[t]{2}{*}{26.58} \\
\hline & 1.41 & 0.2563 & 0.12 & 0.8829 & 0.57 & 0.5712 & \\
\hline \multirow[t]{2}{*}{ LOFO } & 0.61 & $0.546 \quad 18.493$ & 0.66 & 0.52167 .843 & 0.01 & 0.9903 & \multirow[t]{2}{*}{13.732} \\
\hline & 0.29 & 0.7472 & 0.09 & 0.9114 & 0.19 & 0.8282 & \\
\hline
\end{tabular}

Van Valen (1965) menciona que es de esperarse que grupos de medidas tomadas por distintas personas difieran en varianzas, pero no en medias. En este caso, como se muestra en el cuadro 1, existieron diferencias significativas en ambos estadísticos, esto puede deberse precisamente a la práctica, dado que en la persona experimentada (Medidor 1, Cuadro 1) no hubo diferencias ni en medias ni en varianzas, por lo que las diferencias observadas para los medidores dos y tres pueden ser asignables a la falta de experiencia en la toma de medidas.

Además de éste factor existen otros que pueden determinar en forma considerable la variabilidad en la medición de un carácter, uno de los cuales es la relación entre la media y la varianza (Soulé, 1982). Al analizar la correlación entre estos dos estadísticos con el coeficiente de correlación de rangos de Spearman para la primera repetición de cada medidor, se encontró que esta es positiva y significativa para los tres medidores (Medidor $1 r_{s}=0.792$, Medidor $2 r_{s}=0.819$, Medidor $3 r_{s}=0.835$, en los tres casos $\mathrm{n}=20$ y $\mathrm{p}<0.0001$ ); es decir, que entre más grande sea una estructura 0 carácter, mayor variabilidad presenta. Resultados similares obtuvieron Pankakoski et al. (1987) al analizar el error en la medición de cráneos de ratas almizcleras (Ondatra zibethicus), encontrando que este factor explicaba en algunas variables hasta el $45 \%$ de la magnitud del coeficiente de variación, $\mathrm{y}$ al igual que en este estudio, observaron una relación directa entre la media de una variable y su varianza.

Existieron variables cuyas medias difirieron significativamente a pesar de incrementarse la práctica en los medidores inexpertos (repeticiones dos y tres). En 
Cuadro 2. Estimación de tamaños muestrales basados en la varianza de la primera repetición para cada medidor, con un nivel de confianza para la media del $95 \%$ y anchura de intervalo de $5 \%$ del valor de la media de cada variable.

\begin{tabular}{lccc}
\hline Variable & Medidor 1 & Medidor 2 & Medidor 3 \\
\hline LOCR & & & \\
LOCO & 8.408 & 8.553 & 10.197 \\
LOPA & 8.528 & 13.431 & 13.226 \\
LONA & 54.685 & 30.061 & 25.631 \\
LORO & 17.615 & 18.541 & 17.851 \\
ANNAA & 15.968 & 28.479 & 39.822 \\
ANNAP & 9.605 & 27.039 & 41.782 \\
ANCI & 32.964 & 16.887 & 86.964 \\
ANCC & 10.004 & 8.356 & 10.308 \\
ANIN & 13.192 & 9.185 & 10.819 \\
AMF & 68.385 & 124.611 & 35.344 \\
ALCR & 44.267 & 57.571 & 99.039 \\
LOMA & 8.413 & 4.67 & 7.158 \\
ALMA & 14.292 & 25.045 & 32.418 \\
HMMDF & 14.6 & 21.723 & 9.797 \\
HMXD & 9.813 & 11.806 & 12.74 \\
ALRO & 6.453 & 5.58 & 52.763 \\
M3M3 & 36.57 & 34.188 & 11.103 \\
LOFR & 15.032 & 11.26 & 12.944 \\
LOFO & 30.306 & 15.191 & 19.587 \\
\hline
\end{tabular}

contraste, el número de variables significativamente heterocesdásticas disminuyó con la experiencia, ANNAP en la primera y segunda repetición y ninguna variable en la tercera, por lo tanto, se considera que la forma de medir es una fuente importante de variación.

Una forma de minimizar este aspecto es definir con mayor precisión las variables a utilizar. Como ejemplo de esta precisión Bookstein et al. (1985) definen el término "landmark", que es un punto operacionalmente identificable por características de la morfología local y consistente con alguna homología biológica. En contraste el término "pseudolandmark" es un punto que presenta alguna definición operacional creíble, pero no es homólogo de una forma a otra, por ejemplo los puntos extremos de diámetros máximos o mínimos de una estructura. La mayoría de las variables estudiadas en trabajos morfométricos son del tipo "pseudolandmark", en las 
cuales las dimensiones son medidas entre puntos pobremente definidos por "landmarks" biológicos. Por ejemplo, la anchura interorbital no está definida por puntos biológicos homólogos de un individuo a otro (Zeldich et al., 1989).

Sin embargo, Bailey y Byrnes (1990) definieron algunas medidas basadas en "landmarks" en conchas de caracoles, y al compararlos con "pseudolandmarks" encontraron que estructuras tridimensionales complejas pueden ser difíciles de medir con precisión, aún con puntos de referencia bien definidos, por lo que es recomendable en la selección de variables para estudios morfométricos no sólo que los caracteres sean homólogos entre sí, sino que preferentemente sean estructuras fáciles de medir para disminuir este efecto. Además, es recomendable que únicamente una persona se encargue de la medición de la totalidad de los ejemplares que van a ser empleados en el estudio, y que previamente haga al menos dos series de mediciones de muestras estadísticamente representativas (e.g., 20 ejemplares) de la población para estimar el error de medición de la persona, aspecto que se vuelve muy importante, sobre todo en estudios muy finos (e.g., asimetría).

Las consecuencias de la variación intermedidor y de la experiencia pueden ser considerables, sobre todo en estudios de variación intrapoblacional, en los cuales normalmente las muestras son pequeñas, y proporcionan criterios para desarrollar estudios más extensos, como por ejemplo los de variación geográfica, cuyo objetivo es a menudo evaluar el estatus taxonómico de ciertas poblaciones.

Esta variación es también importante en estudios de taxa poco abundantes o pobremente representados en colecciones científicas. Por ejemplo, antes de estudiar la variación geográfica morfométrica en Heteromys gaumeri, Engstrom et al. (1987) analizaron la variación intrapoblacional en 14 medidas de 94 cráneos, entre ellas la anchura interorbital, la cual según este estudio (cuadro 2, Medidor 2) requiere 125 ejemplares para obtener intervalos del $95 \%$ de confianza para la media. Casos similares son los de Williams y Ramírez-Pulido (1984) para Neotomodon alstoni (118 ejemplares), Schmidly (1973) para Peromyscus boyli (154 ejemplares) y de Diersing (1976) para $P$. difficilis (20 ejemplares). La estrategia de medir números estadísticamente representativos de ejemplares y realizar estimaciones de tamaños muestrales mínimos, es un proceso que además de incrementar la confiabilidad de los resultados en este tipo de estudios, también permite optimizar tiempo y los esfuerzos dedicados a esta actividad.

Agradecemos a J. Ramírez-Pulido por permitirnos examinar los ejemplares de la Colección Mastozoológica de la UAM-Iztapalapa. A E. Martínez, M. I. Gómez y G. Palma por su ayuda, a F. Cervantes por el apoyo logístico y a J. Arroyo-C. y dos revisores anónimos por sus valiosas sugerencias. 
Cuadro 3. Diferencias intermedidor en varianzas y en medias, así como varianza debida a fluctuaciones intermedidor (\%VAR). Se empleo únicamente la primera repetición de cada persona. El asterisco indica $\mathrm{P}<0.05$ para la prueba secuencial de Bonferroni.

\begin{tabular}{llllll} 
Variable & \multicolumn{2}{c}{ Varianzas } & \multicolumn{2}{c}{ Medias } & \%VAR \\
& \multicolumn{1}{c}{$\mathrm{F}$} & $\mathrm{P}>\mathrm{F}$ & $\mathrm{F}$ & $\mathrm{P}>\mathrm{F}$ & \\
\hline LOCR & 0.09 & 0.9157 & 0.3 & 0.7405 & 18.85 \\
LOCO & 0.57 & 0.5726 & 2.8 & 0.072 & 42.429 \\
LOPA & 0.75 & 0.4793 & 0.43 & 0.6539 & 23.094 \\
LONA & 0 & 0.9991 & 0.48 & 0.6239 & 24.412 \\
LORO & 0.85 & 0.4353 & 16.67 & $0.0001^{*}$ & 48.544 \\
ANNAA & 1.89 & 0.1642 & 29.13 & $0.0001^{*}$ & 49.156 \\
ANNAP & 7.98 & $0.0012^{*}$ & 20.33 & $0.0001^{*}$ & 48.799 \\
ANCI & 0.08 & 0.9205 & 0.73 & 0.489 & 29.638 \\
ANCC & 0.43 & 0.6505 & 1.39 & 0.2592 & 36.805 \\
ANIN & 1.43 & 0.2501 & 6.07 & 0.0048 & 46.193 \\
AMF & 2.14 & 0.1304 & 2.43 & 0.1007 & 41.456 \\
ALCR & 1.98 & 0.1514 & 2.15 & 0.1288 & 40.575 \\
LOMA & 1.17 & 0.32 & 4.65 & 0.0149 & 45.149 \\
ALMA & 0.78 & 0.4636 & 0.77 & 0.4676 & 30.378 \\
HMMDF & 0.33 & 0.7197 & 0.98 & 0.3826 & 33.144 \\
HMXD & 0.87 & 0.4269 & 1.41 & 0.2565 & 36.88 \\
ALRO & 0.54 & 0.5844 & 4.63 & 0.0153 & 45.122 \\
M3M3 & 0 & 0.9991 & 2.97 & 0.0623 & 42.789 \\
LOFR & 1.36 & 0.2684 & 4.54 & 0.0164 & 45.039 \\
LOFO & 1.18 & 0.3167 & 0.96 & 0.3907 & 32.891 \\
\hline
\end{tabular}

\section{LITERATURA CITADA}

Bookstein, F., B. Chernoff, R. Elder, J. Humpries, G. Smith y R. Strauss. 1985. Morphometrics in evolutionary biology. Academy of Natural Sciences of Philadelphia, Special Publications, 15:1-277.

Bailey, R. C. y J. Byrnes. 1990. A new, old method for assesing measurement error in both univariate and multivariate morphometric studies. Systematic Zoology, 39:124-130.

Daniel, W. D. 1982. Bioestadística: Base para el análisis de las ciencias de la salud. Ed. Limusa, México. EUA.

Diersing, V. E. 1976. An analysis of Peromyscus difficilis from the Mexican-United States boundary area. Proceedings of the Biological Society of Washington, 89:451-466. 
Engstrom, M. D., H. H. Genoways y P. K. Tucker. 1987. Morphological variation, karyology, and systematic relationships of Heteromys gaumery (Rodentia: Heteromyidae). Pp. 289303, in Neotropical mammalogy: Essays in honor of Philip Hershkovitz (B. D. Patterson y R. M. Timm, eds.). Fieldiana: Zoology, New Serie, 39:vii+506.

Lee, J. C. 1982. Accuracy and precision in anuran morphometrics. artifacts of preservation. Systematic Zoology, 31:266-281.

Lee, J. C. 1990. Sources of extraneous variation in the study of meristic characters: the effect of size and of inter-observer variability. Systematic Zoology, 39:3139.

Manly, B. F. J. 1986. Multivariate statistical methods: a primer. Chapman and Hall, New York, EUA.

Pankakoski, E., R. A. Väisänen y K. Nurmi. 1987. Variability of muskrat skulls: measurement error, environmental modification and size allometry. Systematic Zoology, 36:35-51.

Rice, W. R. 1989. Analysing tables of statistical tests. Evolution, 43:223-225.

Santos Moreno, J. A. 1994. Evaluación del uso de las medidas externas estándar en los análisis morfométricos de mamíferos. Anales del Instituto de Biología, Universidad Nacional Autónoma de México, Serie Zoología, 65:275-285.

Schmidly, D. J. 1973. Geographical variation and taxonomy of Peromyscus boylii from Mexico and the southern United States. Journal of Mammalogy, 54:111-130.

Soulé, M. E. 1982. Allomeric variation. I. The theory and some consequences. The American Naturalists, 120:751-764.

Van Valen, L. 1965. Morphological variation and width of ecological niche. The American Naturalists, 99:377-390.

Williams, S. L. y J. Ramírez-Pulido. 1984. Morphometric variation in the volcano mouse, Peromyscus (Neotomodon) alstoni (Mammalia: Cricetidae). Annals of the Carnegie Museum, 53:163-183.

Zeldich, M. L. R. W. Debry y D. O. Straney. 1989. Triangulation-measurement schemes in the multivariate analysis of size and shape. Journal of Mammalogy, 70:571-579. 